\title{
MECHANICAL ANALOGY OF STATEMENT NETWORKS
}

\author{
WOJCIECH CHOLEWA \\ Faculty of Mechanical Engineering \\ Silesian University of Technology, ul. Konarskiego 18a , 44-100 Gliwice, Poland \\ e-mail: wojciech.cholewa@polsl.pl
}

\begin{abstract}
The paper demonstrates briefly the reasoning capabilities in condition monitoring offered by systems based on statement networks. The usefulness of the networks considered results among others from possibilities of their optimization related to the minimization of contradictions between rules acquired from different knowledge sources. A mechanical analogy of such networks introduces an interpretation of statements as material points that are able to move. Dependencies between statements are considered as approximate necessary and approximate sufficient conditions, which are represented by unilateral constraints imposed on the introduced material points. A model of a dynamic statement network can be obtained out of the network consisting of statements represented by material points with assigned masses, where the inertia of statements may be taken into account. The paper introduces a measure of conditional contradictions of statements, which can be used for monitoring knowledge bases in running expert systems.
\end{abstract}

Keywords: statement network, dynamic statement, contradictory rules, belief network.

\section{Introduction}

One of the main achievements of the knowledge domain known as artificial intelligence is the introduction and development of systems able to aid a decision-making process. The subjects of numerous papers are systems which support us when making decisions that facilitate maintaining technical objects sensible. The decisions are reached among other things on the basis of information about past, present and predicted states of an object considered. Such information can be acquired be means of diagnosing and monitoring systems. Diagnosing systems allow us to recognize the current state or a change in this state, considered on the basis of gathered results of observations of an operating object. Monitoring systems make it possible to conduct continuous observation of an object. Results of the observation enable us to recognize changes in technical states.

Two essentially different classes of objects of diagnostic research can be distinguished. Objects that do not consist of elements which undergo gradual wearing belong to the first class. The second class includes objects that consist of such wearing elements. The lack of the parts makes the classification of states easier. In this case, a definition of acceptable wear is not required to be introduced. This does not entail that the design of systems recognizing states of such objects is straightforward, but the lack of elements undergoing wear limits the number of the state classes considered.

The process of drawing inferences about states of objects and their changes can be supported by means of a variety of formalised decision-making and advisory systems. Knowledge-based systems are of great importance. Systems enclosing selected domain knowledge are able to repeatedly apply the knowledge for solving numerous tasks without the direct participation of an expert.

There are three essentially different roles of persons or computer systems taking part in the process of designing and applying knowledge-based systems. The key role is played by the final user, which applies the system as a tool that aids his or her decision-making process dealing with the recognition of a state of the object considered. A domain expert, who is responsible for the knowledge stored in the system, and a knowledge engineer, who is in charge of the manner in which the system operates and the selection of knowledge representation proper for the analysed part of domain knowledge, play two remaining roles. Distinguishing between the roles of domain experts and knowledge engineers is profitable for the development of these systems.

Based on a wide spectrum of papers discussing the principles of operation of such systems, one can conclude 
that a majority of concepts known within the artificial intelligence domain have already been applied. This means that extending the field of feasible solutions of these systems is possible by means of concepts borrowed from other domains. An example is to search for new concepts based on analogies. One of such non-AI domains is mechanics, which suggests that statements representing opinions about the objects can be considered similarly to material points that mutually interact.

One can assume that the location of a material point exemplifies the logical value of a selected statement. The interactions between such points represent cause-effect relationships and associative rules appearing between statements. Moreover, masses assigned to these points let us consider significantly broader capabilities of the discussed models to represent dynamic knowledge bases.

The goal of the paper is to present a concise review of selected systems supporting the diagnostic inference and to discuss the essence of the operation of new systems designed as statement networks, and particularly as dynamic statement networks. The main advantages of statement networks are discussed. Special attention is also paid to the concept of a blackboard which has been known for a long time. At present, the statement networks are extensively studied. Their efficient application in diagnosing and monitoring systems is expected.

\section{Diagnostic expert systems}

In this section a comparison of selected advisory systems to be applied in diagnostic research is presented. Operations of accurate and approximate expert systems are described.

2.1. Expert systems. Expert systems occupy a special place among other systems that aid decision making. An expert system should be able to begin and continue an inference process as well as to carry out operations related to this process in the cases where the user of the system allows or demands such actions. The knowledge considered may include both declarative knowledge in the form of statements about recognized facts and also procedural knowledge related to object operation and general guidelines of the behaviour. The knowledge can be acquired (Moczulski, 2004) from numerous sources. Especially valuable knowledge sources are experts in a given domain, their papers, as well as collections of maintenance data and simulation results. Unfortunately, presently accessible methods have not ensured sufficient effectiveness in the direct acquisition of experts' knowledge yet.

One can consider two categories of expert systems (Cholewa, 2004). They are respectively static systems, operating within a static environment, and dynamic ones, operating within a varying environment and adapted to undertaking tasks in a limited time horizon. A characteristic feature of expert systems is that a sequence of actions making it possible to draw inferences is not established (programmed) in advance while designing the system. Subsequent actions are dynamically established by the inference unit taking into account its present state.

Most expert systems conduct the inference according to the classic two-valued logic. In this case the knowledge base consists of rules represented as follows:

$$
\text { if premise then conclusion, }
$$

where the premise is a logical expression. These rules can constitute chains, in which the conclusion of a selected rule is simultaneously the premise of a successive rule,

$$
\begin{array}{r}
\text { if } \text { premise then }_{\text {conclusion }} \text {, } \\
\text { if } \text { conclusion }_{1} \text { then } \text { conclusion }_{2},
\end{array}
$$

The assumption that the premise of the first rule in the chain is true lets us conjecture that the conclusion and also the premise of the next rule are true. In this case a forward inference, which entails the recognition of conclusions of consecutive rules as true, is possible to be applied. Similarly, the assumption that the conclusion of the last rule in the chain cannot be regarded as true (i.e., it is false) enables us to apply a backward inference, which results in the recognition of current premises and conclusions of preceding rules as false.

Rules can be constituted within sets different from chains. This takes place when premises are complex. Inference in such sets may be conducted by inference engines included within shell systems.

2.2. Varying environment. Expert systems are designed to operate in an environment. A majority of systems being presently applied are static ones, and especially systems that are supposed to act in a fixed environment. The term "fixed environment" does not entail that the environment is unchangeable. It only indicates that for the needs of the system the frozen current state of environment is considered.

It is commonly known that both machinery diagnostics and also process diagnostics require that information about the character of changes in the observed physical quantities, related to the operation of observed machinery or a process, should be taken into account. In order to describe the dynamics of such changes, numerous solutions, introduced ad hoc, have been applied. One of most often employed ways is to extend a set of signals with signal derivatives of the first and second orders. The values of derivatives are carriers of the information about occurring changes. Introducing such additional signal features lets us consider the dynamic environment in a similar way to 
static one. However, there is a lack of universal, commonly accepted methods that would make it possible to design a proper representation of a varying environment.

To discuss systems that include knowledge represented by means of rules, one should pay attention to the fact that these systems make it possible to infer monotonically only. This means that the recognition of new premises, whose logical values were previously unknown, cannot lead to changes in the logical values of previously obtained conclusions. This property is especially inconvenient in diagnostic systems, where conclusions obtained as results of the operation of the system are supposed to be a recognition of a state of an examined object and of where the state of the object may change.

In order to omit limitations resulting from the application of a monotonous inference, a partly nonmonotonous inference is possible. The processes are carried out assuming that the object of interest is considered to be "frozen" at consecutive moments. Results of such processes are a series of conclusions determined independently, where the recognized conclusions can vary within each series.

2.3. Approximate expert systems. The assumption that the knowledge base contains only rules represented in the form (1) is often an excessively strong limitation. Known principles and facts are not exclusive sources of knowledge. The knowledge of a domain considered can be made available in the form of a specialist's inaccurate opinions, uncertain results of object observations or approximate models. An application of such knowledge inaccurate requires that appropriate methods of inference be used. They are characterized by the application of approximate/uncertain rules, premises and conclusions. $\mathrm{Up}$ to now no universal representation of such approximate elements is known. At least two classes of approximate inference methods may be applied. The first one, which will not be discussed, includes methods related to the application of the concept of fuzzy sets and rules (Kuncheva, 2000; Negnevitsky, 2002; Siler and Buckley, 2005). The second class encompasses methods that consist in the application of numerous categories of certainty factors and/or different systems of truth values of elements appearing in the form of premises, conclusions and rules.

A very important and difficult task is to establish a proper interpretation of the measures applied representing uncertainty. They can be interpreted as probabilities of the event that the proposition considered (a given element) is true or as the truth value of this proposition.

The introduction of probabilities lets us apply statistical methods to verify hypotheses. However, a strict application of statistical methods requires numerous assumptions. The possibilities for the verification and validation of the assumptions are very limited. This is a rea- son against the application of probabilities defined on the basis of the event frequency. But such probabilities can be replaced with values established subjectively.

It should be clear that the discussed operations on certainty factors can be a cause of numerous intuitive doubts. The main doubt is that the logical value of a statement (true or false) is often identified with its certainty factor. Different doubts are also related to the assumed models of uncertainty propagation and inaccuracy in the inference unit.

\section{Belief networks}

Approximate expert systems make it possible to apply approximate and/or uncertain rules, premises and conclusions. Truth values and certainty factors of rules and premises are most often estimated on the basis of subjective opinions of designers and specialists. Results of verification tests can be guidelines for essential modifications of previous truth values. The process of the modification of these values is known as the process of system tuning. Important shortcomings of systems based on sets of rules are difficulties of practical execution of such tuning. It is especially difficult in the case of an extensive set of rules.

In systems with knowledge bases in the forms of graphs these difficulties occur not so often. Applications of graphs as models that make it possible to represent knowledge have a long history (Wright, 1934). The most important notable concepts are Markov networks (Isham, 1981; Lauritzen, 1982), often considered in the form of the so-called contingency tables, and Bayesian networks (Pearl, 1988; Jensen, 2001), also known as belief networks. These networks receive wider and wider recognition as efficient tools of approximate inference. A Markov network is a non-directed graph, in which each branch is assigned a symmetric probability of nodes joined by this branch. In contrast to Bayesian networks, Markov ones do not enable us to represent directly knowledge dealing with case-effect relationships, even though they occur between nodes. However, a Markov network can represent cyclic dependencies that a Bayesian network cannot.

A belief network is an acyclic (it does not include cycles) directed graph consisting of nodes and directed branches joining the nodes. A complete set of excluding states is assigned to each node. The values assigned to nodes are vectors which determine distributions of their states and contain values of subjective probabilities interpreted as the degrees of belief that a node is in a given state. Nodes are also characterized by tables that contain the values of conditional probabilities for all elements of the Cartesian product of the states of parent nodes and the states of a node assigned to the table. The tables of conditional probabilities describe relationships between nodes. It is not assumed that the discussed relationships 
are cause-effect dependencies. The inference using a belief network with tables of conditional probabilities and with known values (states) of selected nodes representing results of observations determines unknown values assigned to the remaining nodes. A goal of this procedure is to find a balance within the network. In this case, Bayes' theorem dealing with conditional probabilities is met. Unfortunately, attempts to find global solutions may lead to NP-hard problems. An effective manner is to identify nodes that are conditionally independent. Then local solutions including other nodes, their parents and offspring are possible to be determined. Interesting papers discussing ways of formulating and finding solutions to such tasks are (Jensen, 2001; Pearl, 1988).

\section{Blackboards}

Systems based on the concept of blackboards fulfilled special roles in the development of artificial intelligence. Blackboards are places where announcements (notes, massages) are accessible for receivers. Sources of announcements and their receivers can be users of the systems that consists of blackboards or other systems. Announcements are delivered through their sources to an administrator of the blackboard. The administrator makes a decision about the placement of the notice and about the removal of expired announcements. Receivers observe the blackboard and respond to changes in its state by prescribed operations. They are also able to generate new announcements and send them to the administrator. The concept of the blackboard with announcements considered as knowledge sources was introduced to systems designated to speech interpretation (Engelmore and Morgan, 1988) and then significantly developed (Hayes-Roth, 1995).

There are a number of known manners of practical execution of blackboards. They can be built as hierarchically ordered databases used for the storage of solutions generated by autonomous modules. In order to effectively achieve the best solutions, these modules are entitled to the use (application) of different techniques of inference.

\section{Statements}

Diagnostic expert systems are supposed to aid the recognition of a technical state on the basis of accessible information about the object considered and its operation. The information can be delivered to the systems in the form of various comments, remarks and opinions which describe the observed events, processes and regularities as well as beliefs and conjectures related to the object. In order to apply them as inputs into informative systems, they are represented in the form of logical propositions, occurring as indicative sentences, which can be characterized by different logical values, e.g., true, false or certainty factors and degrees of belief.
In the case of systems based on classical logic, the inference with these sentences is considered in the scope of propositional calculus. They are used as logical variables, which have to be properly interpreted. During an inference these systems start with known premises and pass to conclusions. In accordance with logical relationships between premises and conclusions, the inference can be carried out as deduction or reduction, depending on the direction of logical relationships. Schemes of logical inference can be modified and extended making, e.g., uncertain and inaccurate inference possible. A majority of papers related to the subject focus on a formal part of the inference process, assuming that the interpretation of the logical variables applied does not require that any special operations be employed. Based on the papers dealing with practical attempts to apply various versions of expert systems we can conclude that the problem of a proper interpretation of applied variables, and thus the determination of their meanings, has to be particularly regarded. The assumption that designers of an expert system, authors of its knowledge base and potential users use the same vocabulary and the same principles of conversation, establishing mutual communication, is often groundless and may lead to unintentional effects. It should be considered that information delivered as a result of the operation of the system can be incomprehensible or unclear for its users.

Discussing aspects related to the interpretation, we should take into account a specific role of presented systems. They are advisory systems, and thus such that support decision making. The final decision has to be made by the user of the system. Indirectly, the user takes advantage of the knowledge gathered in the system. One is supposed to ask who is in charge of the final result of all actions and thus the final decision-the user, authors of the knowledge base or designers of the system. Numerous lawyers agree that the full responsibility is borne by the user. Regarding the lack of the possibility of verifying the knowledge base by the user, one should assume that to make the user responsible for all actions, the system is obliged to convince the user that a proposed solution is correct. In order to perform such operations, functions explaining the inference process are included in expert systems.

We should emphasize the usefulness of the concept of statements introduced in (Cholewa, 1996), for making communication with the user easier. Statements can be treated as objects which play roles of logical sentences. They simultaneously contain descriptions of their interpretation, which can be represented in the form of any developed explanations or opinions to a given subject.

The presented approach, dealing with the application of statements, is particularly convenient for systems designated to support technical diagnostics. In this case, problems expected to be solved require that both the interpretation of data values and the interpretation of their changes 
are possible to be determined and delivered to the user.

The word 'statement' has several meanings. Here the statement is an assertion about the recognition of observed facts or assertion representing a given opinion. A statement $s$ can be written down in the form of the pair

$$
s=\langle c, b\rangle,
$$

where $c$ is the statement content, and thus, e.g., an opinion on that a given object is entitled to have a given attribute, whose value is established; $b$ is a (logical) value of the statement. It is defined as the truth factor or degree of belief and concerns the content of the statement.

The discussed statements are approximate since a set of permissible values of statements is not limited to two elements $\{$ true, false $\}$. It is assumed that during the inference the content of a statement is unchangeable, and only its value can vary. The application of a statement makes it possible to attract one's attention to draw a distinction between objective facts and opinions on occurring facts. Such opinions are very often wrongly identified with facts. The definition (3) determines simple statements. It can be extended to the form of (4) that represents a multi-variant statement

$$
s=\left\langle{ }^{n} c,{ }^{n} b\right\rangle,
$$

where ${ }^{n} c$ is an $n$-element list of variants of statement contents, and ${ }^{n} b$ is an $n$-element vector of logical values of the consecutive variants of the contents.

Most often it is assumed that content variants are mutually exclusive.

An example of a multi-variant statement with classical logic values and a number of variants $n=3$ is

$$
\begin{array}{r}
s=\langle\text { "a colour of the sheet is: white }| \text { red } \mid \\
\text { another then white or red", }[0 ; 1 ; 0]\rangle .
\end{array}
$$

The multi-variant statements will be not further discussed. Taking into account (3), these statements can be replaced with proper sets of simple statements, even though this is not fully reasonable.

The application of statements does not directly introduce essential changes in the order and course of the inference process. They simplify the management of the expert systems considered and let us employ a developed help system, containing term explanations, cross-references, and numerous commentaries.

\section{Statement networks}

In statement networks we distinguish between primary and secondary statements. A statement network makes it possible to consider necessary and sufficient approximate conditions. A manner of evaluating inner contradiction, including the conditional one, of the knowledge base represented in the form of a statement network has been proposed. Some possibilities of controlling a range and degree of details of the knowledge acquisition process have been indicated. It is possible to consider local and multi-layer statement networks, which substitute global networks.

6.1. Primary, secondary and output statements. During an inference process being conducted by an expert system, conclusions are recognized or rejected according to the logical values of premises. The discussed fixed set of premises and conclusions is finite and established while building the knowledge base. As the system operates, new conclusions are neither generated nor discovered. This means that inference processes carried out in expert systems are conducted within the so-called closed worlds. Within each system all premises and conclusions appearing in these processes can be replaced with a finite set of statements constituting a network. One may interpret the values of these statements as logical values of replaced premises and conclusions.

In order to extend the initial concept of the blackboard (Engelmore and Morgan, 1988), one can assume that announcements appearing on the blackboard may be replaced by statements constituting an approximate statement network. Changes in the values of statements will initiate a series of actions that cause changes in the values of other statements. This leads to the inference process. A variety of inference rules can be applied. For example, these networks may be considered as special versions of belief networks. This allows us to apply directly inference methods designed for these networks.

The statements considered will belong to classes of primary statements $S_{p}$ or secondary ones $S_{s}$. Primary statements are characterized by values, which are (evidently) independent of the values of other statements and are directly assigned by outer processes (e.g., measurement units or a dialogue with the user). The values of secondary statements depend on the values of other statements appearing within the network and are not directly assigned by an outer processes. One should emphasize that the recognition of dependencies of selected values of statements on other ones does not mean that they are cause-effect relationships. A part of secondary statements can appear in forms of hidden statements, which only play roles of indirect conclusions and which are inaccessible (e.g., invisible) to the user. Secondary statements are applied among other things as auxiliary ones. They are used for the representation of complex expressions consisting of other statements. It is recommended not to use these kinds of statements as hidden ones. An evident representation lets us determine their interpretation which allows us to explain the inference process and makes the interpretation of obtained results easier.

Initial data for the inference process by means of statement networks are sets $B_{p}$. They gather values of 
primary statements that belong to sets $S_{p}$,

$$
B_{p}=\left\{b(x): x \in S_{p}\right\} .
$$

A goal of the inference process is to determine the values $B_{s}$ of all or selected secondary statements, which belong to sets $S_{s}$

$$
B_{s}=\left\{b(x): x \in S_{s}\right\} .
$$

A result of the inference process is presented to the user or an external system in the form of a set $B_{o}$. The set gathers the values of selected statements belonging to a set $S_{o}$ that includes output statements,

$$
B_{o}=\left\{b(x): x \in S_{o}\right\} .
$$

The set $S_{o}$ is determined while taking into account needs and expectations of the user. The set is included in a set of all statements $S$ considered which may gather both primary and secondary statements,

$$
S_{o} \subset S=S_{p} \cup S_{s} .
$$

A division of the set of statements into primary and secondary statements is conditional and not permanent. It depends on presently accessible information coming from outer sources (measurement units, the user's answers etc.).

For example, if an inference process makes a result of measurements characterizing oil temperature as high available, a statement described by the content "oil temperature is high" is considered as a primary one. The lack of such access to the results of measurements in the next inference process makes the oil temperature unknown and this statement belong to the set of secondary statements.

For a given set of primary statements, the inference in statement networks is most often regarded as a task of searching for a balance of values of all discussed statements, while taking into account all dependencies between them.

\subsection{Approximate necessary and sufficient conditions.} Statement networks can be regarded as particular versions of knowledge bases within expert systems. The rules (1) appearing in these systems are represented by means of two classes of conditions: necessary and sufficient ones. If the recognition of the truthfulness of the statement $x$ is always followed by the recognition of the truthfulness of the statement $y$ (but not necessary vice versa), then $x$ is determined as a sufficient condition for $y$. At the same time, $y$ is established as a necessary condition for $x$. If $z$ is simultaneously a necessary and sufficient condition for $y$, then $y$ is the same (i.e., necessary and sufficient) for $x$. The above definitions of necessary and sufficient conditions deal with exact statements that are unambiguously recognized or not. One assumes that in the case of approximate statements which are partially recognized, their values are $b(x)$ and $b(y)$ and can be interpreted as truth values,

$$
b(x) \in[0 ; 1], \quad b(y) \in[0 ; 1],
$$

and the information that $x$ is a sufficient condition for $y$ or that $y$ is a necessary condition for $x$ can be expressed as (Cholewa, 2004)

$$
b(y) \geq b(x) .
$$

Necessary and sufficient conditions determined for exact statements are particular cases of conditions determined for approximate statements according to (11). Considering approximate rules which should be interpreted as approximate necessary and approximate sufficient conditions, we are able to transform (11) into the equivalent approximate form

$$
b(y) \geq b(x)-\delta, \quad \delta \geq 0,
$$

where the parameter $\delta$ is common for all the conditions considered. The non-negative value of this parameter determines an acceptable (permissible) degree of approximation (inaccuracy) of the analysed conditions, where for accurate, exact conditions $\delta=0$.

6.3. Formal correctness of the knowledge base. The requirement of consensus (i.e., the lack of contradictions) among the elements of the knowledge base of expert systems is commonly approved. The formal correctness of the base should be carefully verified while building and maintaining the system.

Identifying contradictory rules within knowledge bases is rather simple when one regards expert systems that interpret exact premises and rules. Unfortunately, this task is difficult in the case of approximate expert systems. The discussed contradiction may be a conditional one, i.e., it may occur for a particular set of recognized statements only. In order to simplify the problem, it is assumed that contradictory rules should be identified during the system operation for an established case, and thus for a known set of recognized premises. Similar processing may be applied to approximate necessary and sufficient conditions. It is based on searching for the minimal value of parameter $\delta$ in (12) that ensures meeting all necessary and sufficient conditions considered.

In this case, the inference process taking into account necessary and sufficient conditions is a search for an equilibrium state in the statement network. It requires that a linear programming problem be solved. Thus, the minimal value of $\delta$ can be also interpreted as an estimator of the contradiction degree. The estimator deals with the set of approximate conditions and assesses the contradiction degree among the elements of the knowledge base. It can be used for permanent monitoring of knowledge bases in running expert systems. 


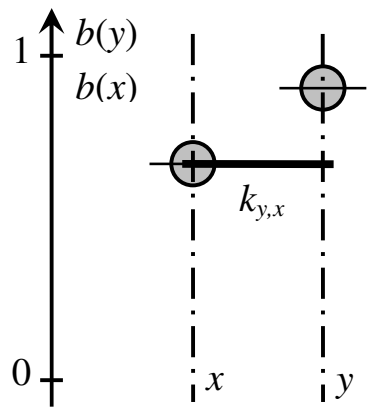

(a)

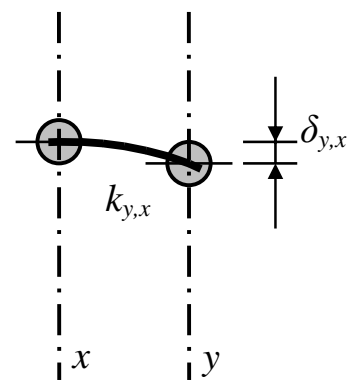

(b)
Fig. 1. Model representing the rule statement $x$ is a sufficient condition for $y$ : (a) the exact condition (11) is meet, (b) the approximate condition (13) is met.

\section{Models of statement networks}

In this section, mechanical analogy of statement networks and the notion of the inertia of statements are introduced.

7.1. Mechanical analogy of statement networks. In order to develop an appropriate method of inference in statement networks taking into account the approximate conditions (12), a model regarded as a mechanical analogy of these networks is considered. We assume that the values of statements included in a statement network are represented as material points that are able to move. The points can be presented graphically. For example, Fig. 1(a) presents the exact condition 111), while Fig. 1(b) presents the approximate condition (13). For example, it may be assumed that the discussed points can move only vertically, whereas the horizontal distances between them are zero. In Fig. 1 the points are shown as "moved horizontally" in order to make their comparison and observation possible. The heights of the point positions correspond to the values of statements (10). Statements unambiguously recognized as true are represented by points located at the level $b(\cdot)$ equal to 1 . Statements recognized as false are located at a level equal to 0 .

Necessary and sufficient conditions are generalizations of the conditions 11 . They are represented by unilateral constraints imposed on the points considered. The constraints correspond to the following inequalities:

$$
b(y) \geq b(x)-\delta_{y, x}, \quad \delta_{y, x} \geq 0 .
$$

The inequality (13) was obtained through a modification of the inequality (12) that consists in the individualization of a parameter $\delta$ and making the assumption that it may take different values $\delta_{y, x}$ for consecutive pairs of statements $(x, y)$ related to the discussed conditions. In Fig. 1 an additional flexible element characterized by the stiffness $k_{y, x}$ is introduced. The element is deformed, as in Fig. 1(b), when the exact conditions (11) cannot be met and only the approximate condition (13) can be considered as partially contradictory to other conditions, e.g., with $\delta_{y, x}>0$. The stiffness $k_{y, x}$ is a parameter that allows distinguishing between the importance of approximate conditions. It may be interpreted as a unit cost of possible contradictions between conditions where the measure of a contradiction degree of a pair of statements is the value of the parameter $\delta_{y, x}$ in (13). The parameters $k_{y, x}$ can take equal values (e.g., 1.0) in the cases where there is no need to distinguish between the importance of conditions. The total cost of all contradictions appearing between elements of the knowledge base can be calculated as the potential energy of the analysed set of points with deformed flexible elements,

$$
E=\sum_{(x, y)} \delta_{y, x} k_{y, x}
$$

The inference within the discussed model of the statement network is to search for an equilibrium state. A minimal potential energy $E$ (14) corresponds to this state. This task can be considered as a classical linear programming problem, which can be represented in the form of a set of inequalities,

$$
\forall(x, y) \in T: \quad b(x)-b(y)-\delta_{y, x} \leq 0,
$$

determining the necessary and sufficient conditions (13) for the set $T$ of selected pairs of statements,

$$
T \subset\left\{(x, y):(x, y) \in(S \times S) \backslash\left(S_{p} \times S_{p}\right) \wedge x \neq y\right\} .
$$

The inequalities (15) represent knowledge gathered in the system.

In the case of this task, restrictions taking into account (10) should be introduced on the values of all secondary statements in the set $S_{s}$

$$
\forall x \in S_{s}: \quad 0 \leq b(x) \leq 1
$$

Additional restrictions result from (13),

$$
\forall(x, y) \in T: \quad \delta_{y, x} \geq 0 .
$$

The restrictions 17 consider only secondary statements, since we assume that due to (10) correctly established values of primary statements meet the condition (17),

$$
\forall x \in S_{p}: \quad 0 \leq b(x) \leq 1 .
$$

It was assumed that the values of primary statements $S_{p}$ and unit costs $k_{y, x}$ are known. Unknown are the values $b(x)$ of secondary statements $x \in S_{s}$, meeting the restrictions (15) and (17), as well as nonnegative parameters $\delta_{y, x}$ minimizing energy $E$, determined according to (14), i.e., such that

$$
E=\sum_{(x, y) \in T} \delta_{y, x} k_{y, x} \rightarrow \min
$$


The determined values $b(x)$ of secondary statements $x \in S_{s}$ exemplify a solution to the task. Unfortunately, the application of the criterion (20) can lead to solutions characterized by several $\delta_{y, x}$ that achieve "high" or even "very high" values while the others achieve "small" values. This means that the conditions (13) corresponding to the parameters $\delta_{y, x}$ whose values are high are excluded from consideration and the remaining ones are considered as exact conditions. It should be suggested that a better strategy will result in a solution that provides a larger number of parameters $\delta_{y, x}$, whose values are "medium", instead of a few "high" values. In this case all conditions are taken into account. The suggested strategy may be carried out by means of a transformation of the linear programming problem with the criterion (20) into a nonlinear programming with the criterion (21) considered together with [15),

$$
E=\sum_{(x, y) \in T}\left(\delta_{y, x}\right)^{2} k_{y, x} \rightarrow \min .
$$

Special attention must be paid to characteristic features of the proposed approach. The introduced model (13) makes it possible for conditionally contradictory rules to simultaneously appear in one knowledge base. The identification of possible contradictions as conditional ones can be performed only for selected states of the statement networks. The cost function (20) or 21) proposed in the paper lets us assess the measure of contradiction degrees for each solution individually. It must be emphasized that the contradiction is estimated as a conditional one. The knowledge base including such contradictions is supposed not to necessary use them while determining other conclusions, and it is possible that from the standpoints of such conclusions the knowledge base considered can be perceived as conditionally compatible. In order to convince the user of the partial correctness of the obtained solution and conditional correctness of the knowledge base applied, information about the value of the cost function (20) or 21) may be shown for each solution. Additionally, it allows the designer of the knowledge base to be indirectly able to identify and monitor a group of contradictory conditions.

7.2. Global, local and multi-layer statement networks. Owing to a large number of statements essential to consider necessary and sufficient conditions between statements, the task of the definition of a statement network directly in the form of a global network for the total set $T$ (16) of the pairs of statements considered can be difficult to perform. In order to simplify the process of representing the acquired knowledge in the form of a statement network, the consideration of a set of local networks that are excerpts of the global one is suggested. To apply this approach, a family of subsets $T_{k}$ of the discussed pairs of statements are determined,

$$
\left\{T_{k} \subset T: k=1, \ldots, K\right\} .
$$

They cover a set $T$, i.e.,

$$
\bigcup_{k=1}^{K} T_{k}=T
$$

Local networks are spanned on subsets $T_{k}$. It was not assumed that these subset are disjoint. This means that local networks defined in this way can overlap and selected statements can appear simultaneously within several local networks. A global network can be defined on the basis of the set of local networks considered. To this end the local networks are assumed to constitute consecutive layers of the global network, which becomes the multi-layer one. A model of the network is determined through assembling local models. Various strategies of assembling the models are possible. They can equalize the participation of component models or statements. The simplest and flexible strategy is that a set $W$ of non-negative weights for the discussed local models can be introduced. The weights are interpreted as measures of importance degrees of consecutive local networks,

$$
W=\left\{w_{k}: k=1, \ldots, K\right\} .
$$

A criterion of optimization for a multi-layer network considered as a global network may be expressed as follows:

$$
E=\sum_{k=1}^{K} w_{k} E_{k} \rightarrow \min ,
$$

where $E_{k}$ is estimated according to (20) or 21) as

$$
E=\sum_{(x, y) \in T_{k}} \delta_{y, x} k_{y, x}
$$

or

$$
E=\sum_{(x, y) \in T_{k}}\left(\delta_{y, x}\right)^{2} k_{y, x} .
$$

The application of local and multi-layer statement networks replacing global networks makes it possible to separately consider selected parts of networks. It allows us to control the degree of granularity in the process of knowledge acquisition for the analysed system.

7.3. Dynamic statements networks. A common feature of all characterized expert systems, belief networks and statement networks is that these systems employ static knowledge bases and do not include memory that would make it possible to take advantages of the history of their actions within the framework of the inference process. A result of their operation is promptly estimated according to changes in input data. The results of the operation of 
belief and statement networks are independent of the order of these changes. This does not totally correspond to inference processes conducted by a human being. While drawing conclusions on the basis of a set of accessible statements, a human being often takes into account not only the values of statements, but also the character of their changes and the periods of time within which a given value is constant. It must be stressed that we often draw conclusions with some delay regarding the moment we were provided with premises. Such a delay also deals with auxiliary conclusions and may have qualitative influence on a complex inference process. It happens especially in when one is able to determine a few different static solutions for the sets of conditions considered. Delays may make technical states characterized by identical symptoms under steady conditions and different symptoms under transient conditions possible to be distinguished. They also allow decreasing the influence of noise on a result of the inference process.

While building systems with statement networks one can introduce modules of data pre-processing. They have the form of low-pass filters and operate between primary statements and data sources corresponding to these statements. Filters average input data and make it possible to obtain a delay. The application of similar filters in order to delay secondary statements is very difficult.

To make the influence on the dynamics of changes in secondary statements possible, one introduces the notion of a dynamic statement. A model of a network including dynamic statements, and thus a model of a dynamic statement network, can be obtained out of a modified statement network represented by a set of conditions 15 . The suggested modification is performed as an assignment of masses to material points representing the statements values, and then the inertia of statements is possible to be taken into account. Inertia has important influence on the time essential to make necessary changes within secondary statements. It was assumed that the value of inertia considered as a statement property is constant. In that network the inference process that is able to propagate changes of statement values is possible to be employed. These changes can be intercepted as dislocations of mutually interacting material points whose masses are determined. The secondary statements of great inertia will change their values more slowly than secondary statements of small inertia.

The proposed model can be further developed with additional introduction of dumping elements. However, it leads to an excessive increase in the complexity of identification tasks of such models.

\section{Conclusions}

Inference processes in diagnostic systems require the support by means of knowledge-based systems. Owing to possibilities of different applications, expert systems with knowledge bases in the form of statement networks are considered to be particularly important. The usefulness of these networks results from possibilities of their optimization related to the minimization of contradictions between different knowledge sources. Among others, independently acquired simple statement networks may be merged into balanced, composite networks. It is rather difficult to merge other kinds of networks, e.g., belief networks, in a similar way. A mechanical analogy of such networks introduces an interpretation of statements as material points that are able to move. A model of a dynamic statement network can be obtained out of a network consisting of statements represented by material points with assigned masses. According to the author, one should expect that dynamic statement networks that make it possible to take into account the inertia of statements will also find interesting applications. Problems dealing with methods of defining as well as applying these networks require further research.

\section{Acknowledgement}

The paper presents a part of the results of research financially supported in the years 2001-2008 with the budget resources of the State Committee for Scientific Research and the Ministry of Science and Higher Education in Poland.

\section{References}

Cholewa W. (1996). Blackboards in technical diagnostics, Proceedings of the Conference on Diagnostics of Industrial Processes, Podkowa Leśna, Poland, pp. 42-47.

Cholewa W. (2004). Expert systems in technical diagnostics, in J. Korbicz, J. M. Kościelny, Z. Kowalczuk and W. Cholewa (Eds.), Fault Diagnosis. Models, Artificial Intelligence, Applications, Springer, Berlin, pp. 591-631.

Engelmore R. and Morgan T. (Eds.) (1988). Blackboard Systems, Addison-Wesley, Reading, MA.

Hayes-Roth B. (1995). An architecture for adaptive intelligent systems, Artificial Intelligence 72(1-2): 329-365.

Isham V. (1981). An introduction to spatial point processes and Markov random fields, International Statistical Review 49(1): 21-43.

Jensen F. V. (2001). Bayesian Networks and Decision Graphs, Springer, New York, NY.

Kuncheva L. (2000). Fuzzy Classifier Design, Physica-Verlag, Heidelberg.

Lauritzen S. L. (1982). Lectures on Contingency Tables, 2nd Edn., University of Aalborg Press, Aalborg.

Moczulski W. (2004). Methods of acquisition of diagnostic knowledge, in J. Korbicz, J. Kościelny, Z. Kowalczuk and W. Cholewa (Eds.), Fault Diagnosis. Models, Artificial Intelligence, Applications, Springer, Berlin, pp. 675-718. 
Negnevitsky M. (2002). Artificial Intelligence. A Guide to Intelligent Systems, Pearson Education Limited, Harlow.

Pearl J. (1988). Probabilistic Reasoning in Intelligent Systems. Networks of Plausible Inference, Morgan Kaufmann, San Mateo, CA.
Siler W. and Buckley J. J. (2005). Fuzzy Expert Systems and Fuzzy Reasoning, Wiley, Hoboken, NJ

Wright S. (1934). The method of path coefficients, The Annals of Mathematical Statistics 5(3): 161-215.

Received: 8 January 2008 Revised: 16 May 2008 